\title{
Serological diagnosis of Mycoplasma pneumoniae infection by using the mimic epitopes
}

\author{
Wenyuan Shi ${ }^{1}$ - Lanhua Zhao ${ }^{2}$ Shengtao $\mathrm{Li}^{1}$ - Guizhen $\mathrm{Xu}^{1}$ - Yanhua Zeng ${ }^{2}$
}

Received: 11 February 2018 / Accepted: 27 May 2018 / Published online: 29 May 2018

๑) Springer Science+Business Media B.V., part of Springer Nature 2018

\begin{abstract}
Nowadays, there is lack of effective serological detection method for Mycoplasma pneumoniae (M. pneumoniae) infection in clinic. In this study, the mimic epitopes of $M$. pneumoniae were screened to evaluate the role in the serodiagnosis of $M$. pneumoniae infection. The M. pneumoniae-positive serum was used as the target for biopanning to phage display random 7-peptide library. The positive phage clones were selected and the DNA were sequenced and analyzed by BLAST. The representative phages were identified using dot immunoblotting and ELISA. The exogenous heptapeptides were synthesized and their reactions with $M$. pneumonia-positive serum were tested by indirect ELISA. Two heptapeptides, namely heptapeptide 1: TVNFKLY and heptapeptide 2: LPQRLRT, were screened out from the randomly selected 40 phages after the four biopanning rounds. They had high homologies to some $M$. pneumoniae antigens. Besides, the representative bacteriophage containing heptapeptide 1 or 2 could react with the M. pneumonia-positive serum. The sensitivities of heptapeptide 1 and heptapeptide 2 for the diagnosis of $M$. pneumoniae infection were 90.1 and $80.0 \%$, respectively, and the specificities were 94.3 and $97.1 \%$, respectively. Therefore the two heptapeptides were the mimic epitopes of M. pneumoniae and might have potential serological diagnosis value for M. pneumoniae infection.
\end{abstract}

Keywords Heptapeptides $\cdot$ Mycoplasma pneumoniae $\cdot$ Mimic epitopes $\cdot$ Serological diagnosis

\section{Introduction}

Mycoplasma pneumoniae (M. pneumoniae) is a very small prokaryote microorganism in the class Mollicutes and it commonly causes infections of the respiratory system (Diaz and Winchell 2016). Generally, M. pneumoniae is of high contagiousness, especially in children, and it is the main pathogen of community-acquired pneumonia (Inchley et al. 2017). But till now, its early diagnosis is difficult because of

Wenyuan Shi and Lanhua Zhao have contributed equally to this work.

Yanhua Zeng

zengyihua21cn@126.com

1 Department of Clinical Laboratory, Chenzhou First People's Hospital, Institute of Translational Medicine, University of South China, Chenzhou 423000, People's Republic of China

2 Institute of Pathogenic Biology, School of Medicine, University of South China, No. 28, West Changsheng Road, Zhengxiang District, Hengyang 421001, People's Republic of China its few unusual symptoms. Thus, early diagnosis of $M$. pneumoniae infection is of extreme importance (Herrera et al. 2016). At present, the diagnosis methods of M.pneumoniae infection mainly include isolation and culture, serological detection (Lee et al. 2017), PCR detection (Li et al. 2017) and physical diagnosis (Kishaba 2016). And there is still lack of high sensitivity of specific antigens for serological diagnosis of M.pneumoniae infection (Wang et al. 2017). Studies have used the C-terminal of the $\mathrm{P} 1$ protein of $M$. pneumoniae, predicted by bioinformatics, as an antigen for serological diagnosis (Xue et al. 2013; Chourasia et al. 2014). Widjaja et al. demonstrate that $\mathrm{P} 90$ and $\mathrm{P} 40$ are main adherence-accessory protein of Mycoplasma Pneumonia, which may be the potential diagnostic antigens (Widjaja et al. 2015). In addition, the N-terminal of P30 protein contains important epitopes (Chang et al. 2011). However, the traditional protein expression and purification methods used in these studies are often of cumbersome operation and high cost, but always of low specificity.

The key sites of antigens for antibodies binding are dominant epitopes or functional epitopes, which generally contain only 5-7 amino acids or monosaccharide residues (Chen and 
Dreskin 2017). And the long peptide epitopes are mostly in the form of spatial conformation. Mimic epitope is a structure in which the sequence is from the discontinuous amino acid of a spatial conformation, and it may have homology to the certain antigens (Larralde and Petrik 2017), or may have no similarity to the antigens at all. Screening of mimic epitopes not only avoids the cumbersome synthesis of long peptides and the purification of recombinant proteins, but also overcomes the low affinity of the long chain amino acids to antibodies due to the helix or folding. Nowadays, mimic epitope screening has been widely used in the identification of diagnostic agents, vaccine development and the determination of protein-specific binding sites (Lebreton et al. 2015).

In 1985, Smith first developed the phage display technology (Smith 1985). This technology can be used to screen high affinity antigen epitopes so as to provide the basis for the serological diagnosis of pathogens and screen of candidate antigens for vaccine (Li et al. 2015; Dong et al. 2017; Liu et al. 2017; Wang and Wang 2017).

In this study, the phage display of the randomized peptide library was used to screen the dominant epitope of M. pneumoniae. The sera of patients suffered from M. pneumoniae infection were used as the targets for screening. Two heptapeptides were obtained, and then synthesized. And an indirect ELISA method (Lee et al. 2016) was used to evaluate its value in serological diagnosis of M. pneumoniae infection.

\section{Materials and methods}

\section{Clinical data of patients}

A total of 83 M. pneumoniae infected patients (from Chenzhou City First People's Hospital) were randomly enrolled in this study. They included 43 males and 40 females, with an average age of 6.2 (range 5-8). M. pneumoniae infection was diagnosed by particle agglutination (PA) test. The titer of M. pneumoniae antibody was higher than $1: 160$. For control, 40 cases of age and gender matched healthy individuals were recruited, including 21 males and 19 females, with an average age of 6.5 (range 5-8). Prior written and informed consent were obtained from every patients and the study was approved by the ethics review board of Chenzhou City First People's Hospital.

\section{Sampling}

Peripheral blood $(2 \mathrm{~mL})$ was collected from each participant from elbow venous. Serum was isolated from peripheral blood with saturated (NH4) ${ }_{2} \mathrm{SO}_{4}$ as previously described (Zeng et al. 2012). The isolated sera were concentrated with ultrafiltration centrifuge tubes (Millipore, Billerica, MA, USA) to remove the inorganic salt ions. The protein concentration was measured by BCA method and stored at $-80^{\circ} \mathrm{C}$.

\section{Screening of phage display random peptide library}

The Ph.D.-7 phage display peptide library, the Escherichia coli ER2738 (the host bacteria of M13 phage), the sequencing primer and horseradish peroxidase (HRP)- labeled antiM13 pIII monoclonal antibody were all from New England Biolabs (NEB, Beverly, MA, USA). The heptapeptide library of phage display was subjected to the four rounds of biopanning as described in previous study (Wang et al. 2016). Briefly, in the first round of panning, the ELISA plates were coated with $100 \mu \mathrm{L}$ of purified serum $(100 \mu \mathrm{g} / \mathrm{mL})$. Then the phages were added and incubated at room temperature for $1 \mathrm{~h}$. After rinsing with $0.1 \%$ TBST, the phages were eluted with $0.2 \mathrm{M}$ Glycine- $\mathrm{HCl}(\mathrm{pH} 2.2)$ and neutralized with $1 \mathrm{M}$ Tris- $\mathrm{HCl}(\mathrm{pH} 9.1)$. After that, reverse adsorption was performed with an empty ELISA plate to exclude the nonspecific binding phages. After the titer determination, $1 \mu \mathrm{L}$ phage was used for amplification. The basic steps of the second to fourth round of biopanning were similar to those in the first round, except that the concentration of the coating serum was reduced to $60 \mu \mathrm{g} / \mathrm{mL}$ and the concentration of the rinsing solution was increased to $0.3 \%$ TBST to minimize the nonspecific adsorption. Finally, the yield of each round of panning was calculated based on the output and the input of phages.

\section{DNA sequencing and analysis}

The screened phages were plated and cultured. Then, 40 colonies were randomly selected from the plates with less than 100 plaques and amplified. The phage single-stranded DNA was extracted from these colonies with sodium iodide method and sequenced by Sangon Biotech Co., Ltd., (Shanghai, China). The exogenous DNA sequence of the phage display was deduced and translated into amino acid sequences for BLAST alignment.

\section{Synthesis and purification of the two heptapeptides}

The screened heptapeptides (heptapeptide 1 and heptapeptide 2) was synthesized by the solid phase method in Shanghai Jier Biochemical Company (Shanghai, China). The peptides were purified by reversed phase high performance liquid chromate- graphy (HPLC) and then identified by mass spectrometry. 


\section{ELISA}

ELISA was performed as previously described (Wang et al. 2016). For the binding of phages to M. pneumoniaepositive serum, the ELISA plate was coated with $200 \mu \mathrm{L}$ purified positive serum $(100 \mu \mathrm{g} / \mathrm{mL})$ at $4{ }^{\circ} \mathrm{C}$ overnight, while a negative serum control group and a BSA control group were set up. Followed by blocking with 5\% skim milk for $3 \mathrm{~h}$, the plates were rinsed for five times with $0.5 \%$ PBST. Then $50 \mu \mathrm{L}$ of the phages were added into the plates and incubated at $37{ }^{\circ} \mathrm{C}$ for $2 \mathrm{~h}$. After rinsing with $0.5 \%$ PBST for five times, HRP-labeled anti-M13 pIII monoclonal antibody (NEB, Beverly, MA, USA) was added and incubated at $37{ }^{\circ} \mathrm{C}$ for $15 \mathrm{~min}$.

For the binding of $M$. pneumoniae-positive serum to the purified heptapeptides, the ELISA plate was coated with $200 \mu \mathrm{L}$ heptapeptides $(20 \mu \mathrm{g} / \mathrm{mL})$ at $4{ }^{\circ} \mathrm{C}$ overnight. The plate was then blocked with 5\% skim milk for $3 \mathrm{~h}$ at room temperature. Then, $50 \mu \mathrm{L}$ of diluted serum (1:10) was added and incubated at $37{ }^{\circ} \mathrm{C}$ for $45 \mathrm{~min}$. After rinsing with $0.5 \%$ PBST for 5 times, the HRP-conjugated goat anti-human IgG antibody (1: 5000) (Cat \# 62-8420, Invitrogen, USA) was added and incubated at $37^{\circ} \mathrm{C}$ for $30 \mathrm{~min}$. After rinsing, the reaction was terminated with coloration for $10 \mathrm{~min}$. Then the OD value at a wavelength of $450 \mathrm{~nm}$ was measured by a microplate reader (Tecan, Infinite F50, Swiss).

\section{Dot blot analysis}

The binding of phages to positive sera was further verified by dot blotting experiment (Wang et al. 2016). Briefly, $1 \mu \mathrm{L}$ of the amplified phage supernatant was added into the small square on the PVDF membrane. M.pneumoniaepositive serum was used as positive control. At the same time, BSA, M.pneumoniae-negative serum and wild type vcsM13 phage were used as the negative controls. After the liquid was absorbed by PVDF membrane, the PVDF membrane was blocked overnight at $4{ }^{\circ} \mathrm{C}$, and then rinsed four times with TBST. Then M.pneumoniae-positive sera at 1:100 dilution, which was used as the primary antibody, and negative controls were added and incubated at room temperature for $2 \mathrm{~h}$. After rinsing with TBST for 4 times and $30 \mathrm{~min}$ each time, the samples were incubated with HRP-labeled goat anti-human IgG (1:5000 dilution; Cat \# 62-8420, Invitrogen, USA), which served as the secondary antibody, at room temperature for $1 \mathrm{~h}$. The membrane was then rinsed 4 times with TBST, 20 min each time. Finally, dot blot was developed with electrochemiluminescence (ECL) using a chemiluminescence imaging system.

\section{Statistical analysis}

The sensitivity and specificity of the ELISA method were analyzed as follows: Sensitivity $=$ true positive number/ (true positive number + false negative number) $\times 100 \%$, and, specificity $=$ true negative number/(true negative number + false positive number $) \times 100 \%$ (16). The receiver operating characteristic curve (ROC) was plotted with GraphPad Prism software. The area under the ROC curve (AUC) was calculated. When the AUC $<0.5$, it indicates that the ELISA results are not reliable; when $0.5<\mathrm{AUC}<0.7$, it suggests that the credibility of the ELISA results are low; when $0.7<\mathrm{AUC}<0.9$, it means that the ELISA accuracy is higher and that the results have a higher credibility; when AUC $>0.9$, it indicates that the accuracy is very high. In addition, we also used MedCal to calculate the sensitivity and specificity of ELISA, and the nearest point from the upper left corner of the ROC curve $(100,0)$ was used as the criterion for the ELISA diagnosis of $M$. pneumoniae infection.

\section{Results}

\section{The specific phages are enriched by the four rounds of biopanning}

In order to enrich the phages that specifically bind to the $M$. pneumoniae-positive sera from the randomized heptapeptide library, four rounds of the phage display screening were performed. The results showed that the phage yield gradually increased with the screening rounds (Table 1), indicating that the phages specifically binding to M. pneumoniae-specific serum have been greatly enriched.

\section{Analysis of the DNA sequence and peptide sequence of the screened phages}

In order to determine the DNA sequence of the screened phages, 40 monoclonal phages were randomly selected and DNA sequencing was performed. The results of agarose gel electrophoresis for 20 samples of the extracted DNA were shown in Fig. 1. The single strand DNA of M13 phage was about $6.4 \mathrm{~Kb}$, thus the DNA band located on about $3 \mathrm{~Kb}$

Table 1 The ratio of output and input by affinity screening

\begin{tabular}{|c|c|c|c|c|}
\hline Screening rounds & First & Second & Third & Fourth \\
\hline Input phage titer (pfu) & $4 \times 10^{10}$ & $4 \times 10^{10}$ & $4 \times 10^{10}$ & $4 \times 10^{10}$ \\
\hline $\begin{array}{l}\text { Output phage titer } \\
\text { (pfu) }\end{array}$ & $2.0 \times 10^{2}$ & $6.7 \times 10^{3}$ & $9.1 \times 10^{4}$ & $6.7 \times 10^{5}$ \\
\hline Productive rate $(\%)$ & $5.0 \times 10^{-9}$ & $1.7 \times 10^{-7}$ & $2.3 \times 10^{-6}$ & $1.7 \times 10^{-5}$ \\
\hline
\end{tabular}


Fig. 1 Analysis of single stranded DNA by agarose gel electrophoresis. $M$ DNA-ladder; lane 1-20: single-stranded DNA extracted from randomly selected phage clones

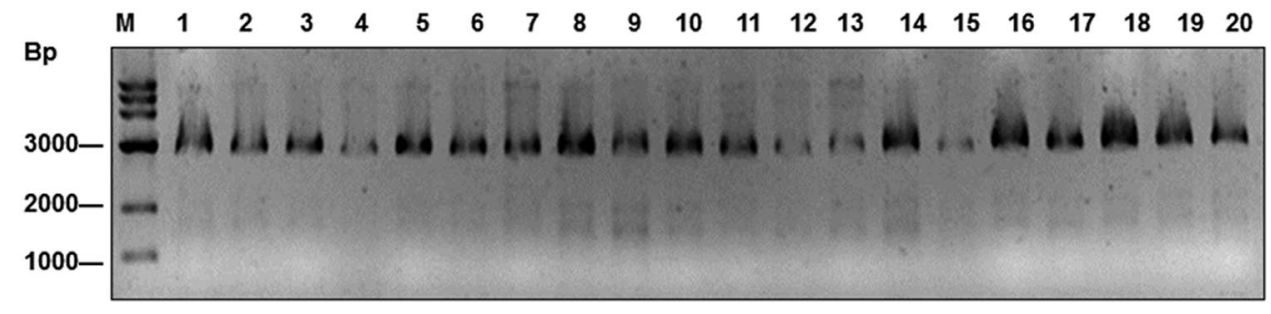

\begin{tabular}{llllllllr}
\hline Phage number & \multicolumn{2}{l}{ Exogenous amino acid sequence exhibited by phages } & & Repeats \\
\hline 1 & $\mathrm{~T}$ & $\mathrm{~V}$ & $\mathrm{~N}$ & $\mathrm{~F}$ & $\mathrm{~K}$ & $\mathrm{~L}$ & $\mathrm{Y}$ & 20 \\
2 & $\mathrm{~L}$ & $\mathrm{P}$ & $\mathrm{Q}$ & $\mathrm{R}$ & $\mathrm{L}$ & $\mathrm{R}$ & $\mathrm{T}$ & 5 \\
3 & $\mathrm{R}$ & $\mathrm{S}$ & $\mathrm{L}$ & $\mathrm{R}$ & $\mathrm{Q}$ & $\mathrm{R}$ & $\mathrm{Y}$ & 2 \\
4 & $\mathrm{H}$ & $\mathrm{R}$ & $\mathrm{R}$ & $\mathrm{Q}$ & $\mathrm{M}$ & $\mathrm{P}$ & $\mathrm{L}$ & 2 \\
5 & $\mathrm{~T}$ & $\mathrm{P}$ & $\mathrm{K}$ & $\mathrm{L}$ & $\mathrm{O}$ & $\mathrm{R}$ & $\mathrm{M}$ & 1 \\
\hline
\end{tabular}

Table 2 Screened polypeptides after phage sequencing

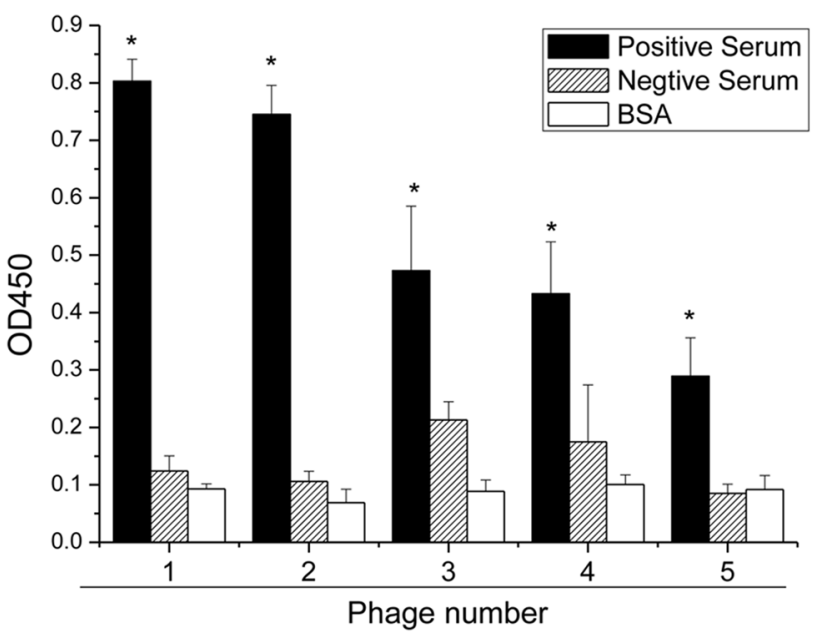

Fig. 2 ELISA analysis of the screened 5 phage clones. The ELISA plates were coated with $M$. pneumoniae-positive serum, negative serum or BSA, respectively. The five representative phages were added and incubated $2 \mathrm{~h}$ at $37{ }^{\circ} \mathrm{C}$ after blocking. And then HRPlabeled anti-M13 pIII monoclonal antibody was added and incubated for $15 \mathrm{~min}$ at $37^{\circ} \mathrm{C}$. The OD450 value was measured. Phage $1-5$ represents the five representative phages. ${ }^{*} p<0.05$, compared with negative serum or BSA

were clear spots in the places with representative phage 1 and 2 or the positive control. However, there was no spot for the negative control. These results demonstrated that the representative phages 1 and 2 could specifically bind to the positive sera from $M$. pneumoniae infected patients.

\section{Both heptapeptide 1 and 2 have high homology to the M. pneumoniae proteins}

To analyze the homology of the two heptapeptides with the M. pneumoniae proteins, we performed BLAST analysis 


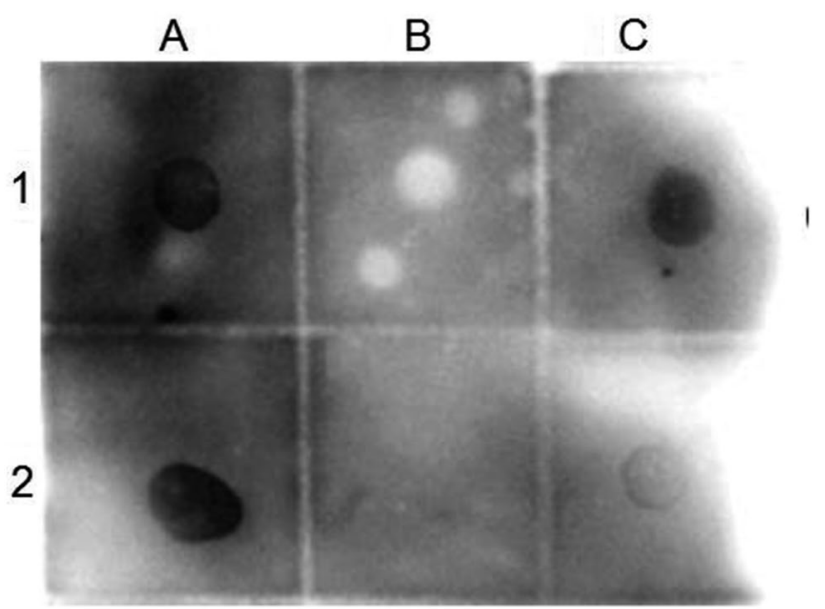

Fig. 3 Phage plaque immunoblot assay. The representative phages were added on the small square of the PVDF membrane. The PVDF membrane was then incubated with the purified M.pneumoniaepositive serum. BSA, M. pneumoniae-negative serum and wild type vcsM13 phage were used as negative controls. Al representative phage $1, B 1$ blank control, $C 1$ purified $M$. pneumoniae positive serum, $A 2$ representative phage $2, B 2$ wild type M13 phage control, $C 2$ purified $M$. pneumoniae negative serum control

respectively. The results are shown in Tables 3 and 4 . The heptapeptide 1 had a high homology with 21 proteins of $M$. pneumoniae, especially with the adenine methyltransferase of M. pneumoniae at the 176-180 position (NFKLY), which were exactly the same (5/5) (Table 3 ). Besides, heptapeptide
1 also had $86 \%$ homology with amino acids at $172-178$ of $\alpha / \beta$ hydrolase (6/7) (Table 3). Meanwhile, heptapeptide 2 had high homology with $8 \mathrm{M}$. pneumoniae proteins. There was $80 \%$ homology between heptapeptide 2 and ribonuclease $\mathrm{R}$ at position 337-341 and four amino acids was exactly identical to ribonuclease R (Table 4). However, neither of these two heptapeptides had a sequence that was identical to that of M.pneumoniae. This data suggest that the two heptapeptides may be the mimic epitopes of M. pneumoniae .

\section{The screened polypeptides synthesized by solid-phase synthetic can specifically bind to $M$. pneumoniae-positive sera}

The two heptapeptides were synthesized by solid phase method and purified by RP-HPLC. And the synthesized peptides were then identified by mass spectrometry. The purity of synthesized two heptapeptides was higher than $95 \%$. In order to verify that the specific binding of phages to $M$. pneumoniae infected sera was caused by exogenous heptapeptides itself, but not by the phages, we further performed ELISA. As shown in Fig. 4a and b, the OD values of $M$. pneumoniae positive sera were obviously higher than those of $M$. pneumoniae negative sera for heptapeptide 1 and 2. The sensitivity of the indirect ELISA method based on heptapeptide 1 was $90.1 \%$, the specificity was $94.3 \%$, and the AUC was 0.96 (Fig. 4c) (Table 5). While the sensitivity of the indirect ELISA method based on heptapeptide
Table 3 BLAST results for the heptapeptide 1 displayed by phage 1

\begin{tabular}{lllll}
\hline Matched protein & Sequence ID & Query & Subject & Identities (\%) \\
\hline Adenine-specific methyltransferase & WP_010874555.1 & $3-7$ & $176-180$ & $5 / 5(100 \%)$ \\
Alpha/beta hydrolase & WP_010874829.1 & $2-7$ & $172-178$ & $6 / 7(86 \%)$ \\
DNA polymerase III subunit alpha & WP_058158328.1 & $4-7$ & $1285-1288$ & $4 / 4(100 \%)$ \\
Hypothetical protein & WP_086132312.1 & $4-7$ & $767-770$ & $4 / 4(100 \%)$ \\
ATP-dependent chaperone ClpB & WP_086131711.1 & $2-5$ & $568-571$ & $4 / 4(100 \%)$ \\
ATP-dependent chaperone ClpB & WP_086131711.1 & $2-5$ & $568-571$ & $4 / 4(100 \%)$ \\
Clp protease ClpB & 054173792.1 & $2-5$ & $569-572$ & $4 / 4(100 \%)$ \\
Chaperone protein ClpB & WP_010874888.1 & $2-5$ & $568-571$ & $4 / 4(100 \%)$ \\
Membrane protein & WP_038534343.1 & $2-5$ & $511-514$ & $4 / 4(100 \%)$ \\
Metal-dependent phosphohydrolase & WP_054175390.1 & $2-5$ & $318-321$ & $4 / 4(100 \%)$ \\
Ribonuclease Y & WP_010874626.1 & $2-5$ & $318-321$ & $4 / 4(100 \%)$ \\
Hypothetical protein & WP_086131702.1 & $3-6$ & $700-703$ & $4 / 4(100 \%)$ \\
Hypothetical protein & WP_080998166.1 & $3-6$ & $635-638$ & $4 / 4(100 \%)$ \\
Cytadherence-related protein & WP_014325371.1 & $3-6$ & $633-636$ & $4 / 4(100 \%)$ \\
MgpC protein & WP_014574889.1 & $3-6$ & $633-636$ & $4 / 4(100 \%)$ \\
ATP-dependent helicase & WP_053344065.1 & $3-6$ & $126-129$ & $4 / 4(100 \%)$ \\
PTS system, glucose-specific IIABC component & BAV20433.1 & $3-6$ & $912-915$ & $4 / 4(100 \%)$ \\
Cytadhesin MgpC-like protein & WP_041359961.1 & $3-6$ & $414-417$ & $4 / 4(100 \%)$ \\
Cytadherence-related protein ORF6 homolog & BAV19569.1 & $3-6$ & $55-58$ & $4 / 4(100 \%)$ \\
DNA polymerase III subunit alpha & WP_086132698.1 & $1-6$ & $836-841$ & $4 / 6(67 \%)$ \\
Hypothetical protein & WP_053344021.1 & $1-6$ & $127-132$ & $4 / 4(67 \%)$ \\
\hline & & & & \\
\hline
\end{tabular}


Table 4 BLAST results for the heptapeptide 2 displayed by phage 2

\begin{tabular}{lllll}
\hline Matched protein & Sequence ID & Query & Subject & Identities (\%) \\
\hline Ribonuclease R & WP_054173846 & $1-5$ & $337-341$ & $4 / 5(80 \%)$ \\
Threonine-tRNA Ligase & WP_010874910.1 & $1-4$ & $277-280$ & $4 / 4(100 \%)$ \\
IS21-family transposase, partial & WP_081038395.1 & $2-5$ & $384-387$ & $4 / 4(100 \%)$ \\
Hypothetical protein & WP_010874754.1 & $2-5$ & $193-196$ & $4 / 4(100 \%)$ \\
Hypothetical protein & WP_053344140.1 & $4-7$ & $126-129$ & $4 / 4(100 \%)$ \\
Haloacid dehalogenase & WP_010874739 & $4-7$ & $126-129$ & $4 / 4(100 \%)$ \\
ABC transporter ATP-binding protein & WP_010874772 & $2-6$ & $119-123$ & $4 / 5(80 \%)$ \\
Excinuclease ABC subunit B & WP_058157860.1 & $3-7$ & $573-577$ & $4 / 5(80 \%)$
\end{tabular}
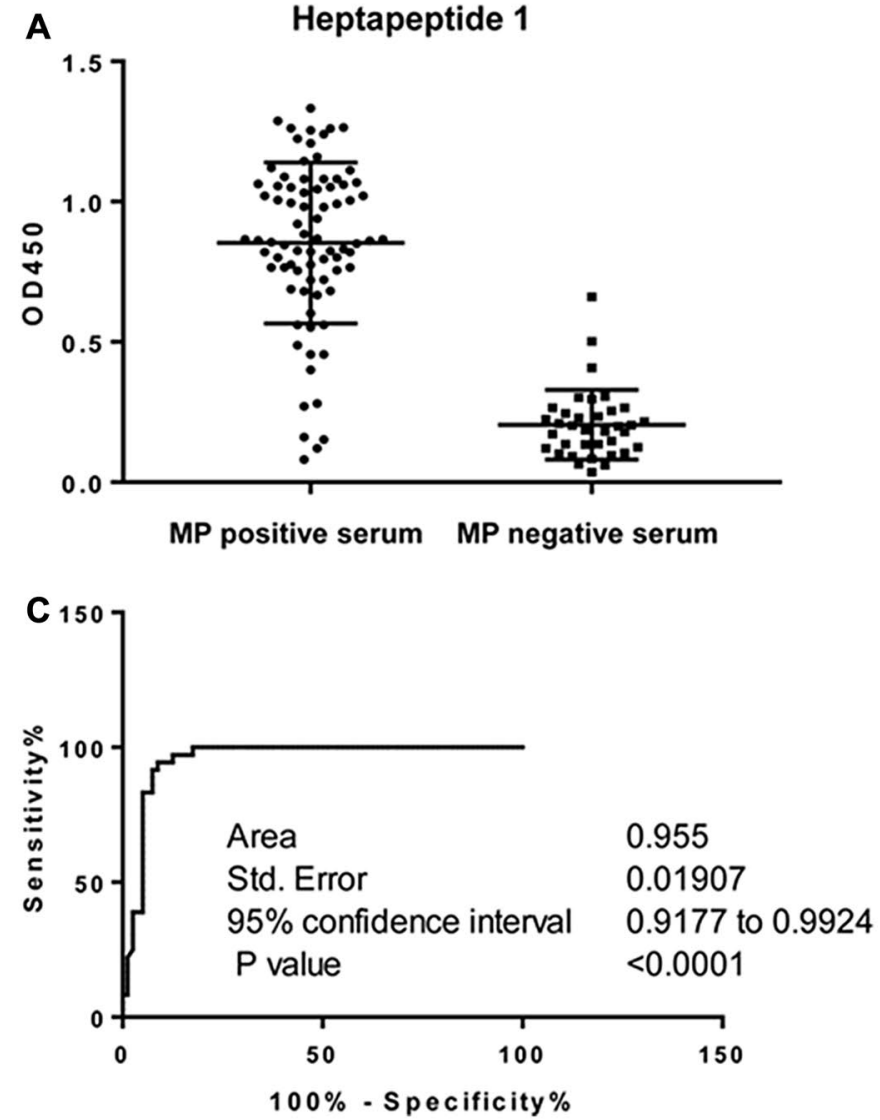

Fig. 4 ELISA analysis of two heptapeptides with different $M$. pneumoniae serum and the ROC curve. ELISA reaction of two heptapeptides to different pneumoniae serum was analyzed and the receiver operating characteristics (ROC) curve was evaluated. The ELISA plates were coated with the corresponding heptapeptide. Then $M$. pneumoniae sera, including 80 positive sera and 36 negative sera, were added and incubated $45 \mathrm{~min}$ at $37{ }^{\circ} \mathrm{C}$ followed by incubating
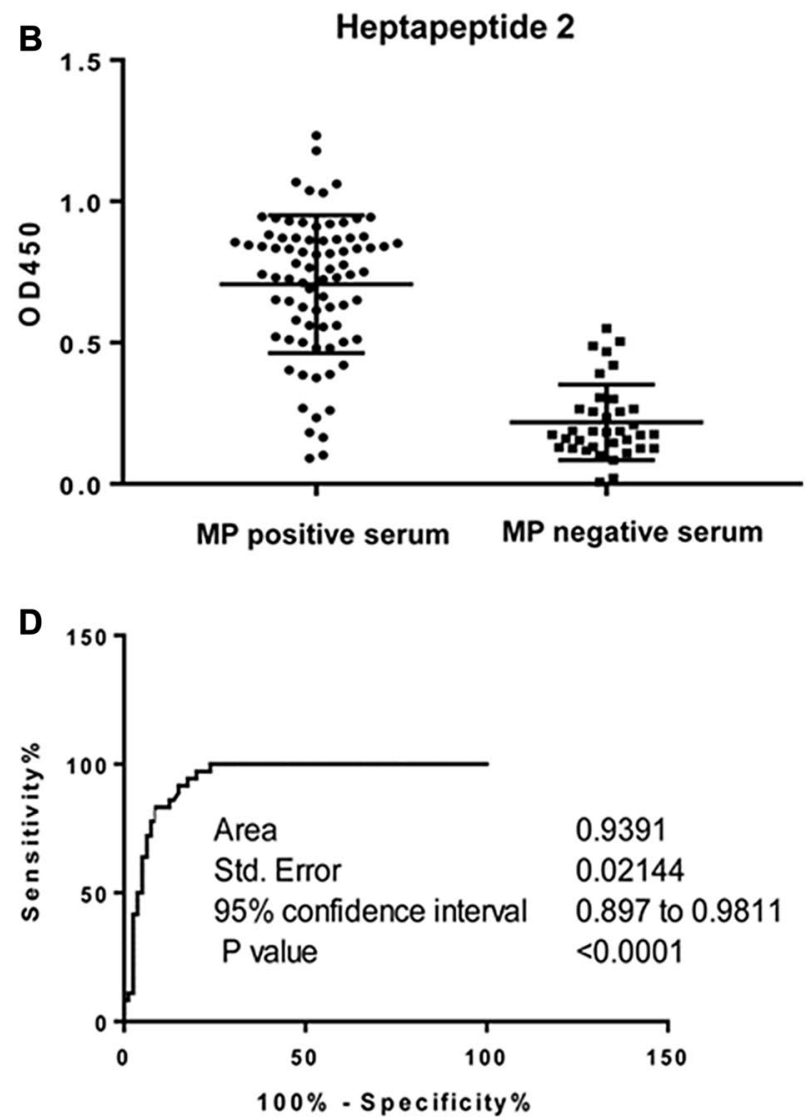

30 min with the HRP-conjugated goat anti-human IgG antibody. The area under the curve, standard error and 95\% confidence intervals $(95 \% \mathrm{CI})$ and $\mathrm{p}$ value were shown in $\mathbf{c}$ and $\mathbf{d}$. a The OD value of heptapeptide 1 to different $M$. pneumoniae serum. b The OD value of heptapeptide 2 to different $M$. pneumoniae serum. $\mathbf{c}$ ROC curve of heptapeptide 1 to different $M$. pneumoniae serum. d ROC curve of heptapeptide 2 to different $M$. pneumoniae serum
2 was $80.00 \%$, the specificity was $97.1 \%$, and the AUC was 0.94 (Fig. 4d) (Table 5). The AUC values of both heptapeptides were greater than 0.9, indicating that both the accuracy and reliability of ELISA are high and may have potential clinical diagnostic value.

\section{Discussion}

At present, epitope screening methods include bioinformatics prediction, high-throughput protein chip 
Table 5 ELISA reaction results of the synthesized heptapeptide

\begin{tabular}{lllllll}
\hline Heptapeptide & AUC & $P$ value & Threshold & You Den Index & $\begin{array}{l}\text { Sensitivity (\%) } \\
\text { M.P positive } \\
\text { serum }(\mathrm{n}=80)\end{array}$ & $\begin{array}{l}\text { Specificity (\%) } \\
\text { M.P negative } \\
\text { serum (n=36) }\end{array}$ \\
\hline 1 & $0.96 \pm 0.019$ & $<0.0001$ & 0.408 & 0.84 & 90.1 & 94.3 \\
2 & $0.94 \pm 0.021$ & $<0.0001$ & 0.504 & 0.77 & 80.00 & 97.1 \\
\hline
\end{tabular}

technology, and peptide library screening (Du et al. 2015; Muthusamy et al. 2016; Xiao et al. 2016). However, the recombinant protein expressed by bioinformatics prediction is generally of dozens or even hundreds of amino acids and often carries an expression tag (Hostetler et al. 2015), whereas the costs of protein chip are high and the three-dimensional structure of protein cannot be maintained. Comparatively speaking, phage display random peptide libraries can screen epitopes that are expressed in natural environments and do not contain extra amino acids (Hostetler et al. 2015). The mimic epitopes screened using phage display peptide library technique can simulate both linear epitopes and conformational epitopes and have been widely used in the development of diagnostic antigens or vaccines (Cui et al. 2016) for many diseases, such as Leishmania (Costa et al. 2014), Hepatitis E virus (HEV) (Larralde and Petrik 2017), Microsporidia (Santos et al. 2012), Rabies virus (Yang et al. 2013), and Chlamydia pneumoniae.

The advantage of phage display technology is that the mimic epitopes are of strong immunogenicity and can be obtained without the knowledge of the antigen structure (Chen and Dreskin 2017). It can also help to avoid the peptides not binding to antibodies in vitro because of the conformational epitopes (Chen and Dreskin 2017). At the same time, phage display technology is easy to operate, and can make up for defects of antigen toxicity and high costs (Pinschewer 2017). In this study, a total of four rounds of biological panning were performed to obtain the specific binding phages. In accordance with previous study (Wang and Wang 2017), highly specific phage clones were enriched by gradually decreasing the coating concentration of serum antigens, as well as increasing the concentration of Tween in the rinsing solution and reducing the incubation time or prolonging the elution time. In addition, the serum from several M. pneumoniae-infected patients contained widely distributed and varied polyclonal antibodies. Thus it could ensure that the epitopes selected by the phage display peptide library had broad spectrum. Our results also showed that there was high binding specificity between the screened phages and the M.pneumoniae-positive serum.

After DNA sequencing and amino acid translation, two heptapeptides with the highest repetition were obtained. After further blasting, we found that the mimotope heptapeptide 1 obtained in this study had high homology with $\alpha / \beta$-hydrolase and adenine-specific methyltransferase of M. pneumoniae. Generally, the $\alpha / \beta$-hydrolases are involved in metabolism and biosynthesis, which can degrade host cell surface lipids, and are associated with the virulence invasion of pathogens (Johnson 2017). The screened heptapeptide 1 was also of $86 \%$ homology to the amino acids $172-178$ of $M$. pneumoniae $\alpha / \beta$-hydrolase. The hydrolase may form a conformational epitope to bind to the antibody (Singh et al. 2017). This may be the reason that the heptapeptide 1 is not exactly the same as $\alpha / \beta$-hydrolase. Similarly, the BLAST results showed that the heptapeptide 2 was identical to the four amino acids of the threonyl tRNA ligase (Zhou et al. 2016) and the IS21 family of transposase.

The indirect ELISA method based on the two mimic peptides screened in this study both have high sensitivity and specificity, and they all have a clinical value for serological diagnosis of $M$. pneumoniae infection. However, this study is limited in that the M. pneumoniae positive sera were collected by particle agglutination test and that the mimic epitopes screened in this study may be not pathogen-specific, although they had various $M$. pneumoniae antigenic homologies. Thus, the sensitivity and specificity in the present method were lower than those in established serologic tests as shown in this study. Additionally, we did not use the paired, serologically confirmed samples that showed an IgM seroconversion or IgM antibody titer increase.

For the further studies, we will express the proteins that have high homology to these peptides, and then test these recombinant proteins in the serological diagnosis of $M$. pneumoniae infection. In addition, we will further expand serum samples to get more statistically significant of the sensitivity and specificity for the ELISA test. In summary, we established an indirect ELISA method using the purified heptapeptides as the target to detect M. pneumoniae-infected positive serum. We found that this method had high sensitivity and specificity, and potential serological diagnosis value for M. pneumoniae infection.

In conclusion, two heptapeptides (heptapeptide 1: TVNFKLY and heptapeptide 2: LPQRLRT) with high homology to some of the $M$. pneumoniae antigens have been identified after screening the phage display random peptide library. They can specifically bind with the serum from M.pneumoniae-infected patients with high affinity. Our findings demonstrate that the two identified heptapeptides may 
have potential serological diagnosis value for M. pneumoniae infection.

Acknowledgements This work was supported by National natural science foundation of China (No. 31370207), Chenzhou municipal bureau of science and technology (No. czkj2016045) and the project of Chenzhou First People's Hospital (No. N2014-001) and the scientific Research Found of Hunan Provincial Education Department (17C1383).

\section{Compliance with ethical standards}

Conflict of interest All authors declare no financial and non-financial competing interests.

\section{References}

Chang HY, Prince OA, Sheppard ES, Krause DC (2011) Processing is required for a fully functional protein P30 in Mycoplasma pneumoniae gliding and cytadherence. J Bacteriol 193:5841-5846. https://doi.org/10.1128/jb.00104-11

Chen X, Dreskin SC (2017) Application of phage peptide display technology for the study of food allergen epitopes. Mol Nutr Food Res. https://doi.org/10.1002/mnfr.201600568

Chourasia BK, Chaudhry R, Malhotra P (2014) Delineation of immunodominant and cytadherence segment(s) of Mycoplasma pneumoniae P1 gene. BMC Microbiol 14:108. https://doi. org/10.1186/1471-2180-14-108

Costa LE et al (2014) Subtractive phage display selection from canine visceral leishmaniasis identifies novel epitopes that mimic Leishmania infantum antigens with potential serodiagnosis applications. Clin Vaccine Immunol 21:96-106. https://doi.org/10.1128/ cvi.00583-13

Cui Y, Yu L, Zhou Y, Yang L, Zhang C (2016) Mimotope identification of dust mite allergen Der $\mathrm{f} 5$ using phagedisplayed random peptide libraries. Mol Med Rep 14:4816-4822. https://doi.org/10.3892/ mmr.2016.5848

Diaz MH, Winchell JM (2016) The evolution of advanced molecular diagnostics for the detection and characterization of Mycoplasma pneumoniae. Front Microbiol 7:232. https://doi.org/10.3389/fmicb .2016 .00232

Dong Y, Lv W, Lin Z (2017) Value of serum Mycoplasma pneumoniae immunoglobulin in the diagnosis of mycoplasma-related pneumonia in newborns. Exp Ther Med 14:1445-1449. https://doi. org/10.3892/etm.2017.4654

$\mathrm{Du} \mathrm{H}$ et al (2015) Screening hybridomas for anabolic androgenic steroids by steroid analog antigen microarray. Bioanalysis 7:12011209. https://doi.org/10.4155/bio.15.67

Herrera M, Aguilar YA, Rueda ZV, Muskus C, Velez LA (2016) Comparison of serological methods with PCR-based methods for the diagnosis of community-acquired pneumonia caused by atypical bacteria. J Negat Results Biomed 15:3. https://doi.org/10.1186/ s12952-016-0047-y

Hostetler JB, Sharma S, Bartholdson SJ, Wright GJ, Fairhurst RM, Rayner JC (2015) A library of plasmodium vivax recombinant merozoite proteins reveals new vaccine candidates and proteinprotein interactions. PLoS Neg1 Trop Dis 9:e0004264. https://doi. org/10.1371/journal.pntd.0004264

Inchley CS, Berg AS, Vahdani Benam A, Kvissel AK, Leegaard TM, Nakstad B (2017) Mycoplasma pneumoniae: a cross-sectional population-based comparison of disease severity in preschool and school-age children. Pediatr Infect Dis J 36:930-936. https://doi. org/10.1097/inf.0000000000001628

Johnson G (2017) The alpha/beta hydrolase fold proteins of Mycobacterium tuberculosis, with reference to their contribution to virulence. Curr Protein Pept Sci 18:190-210. https://doi. org/10.2174/1389203717666160729093515

Kishaba T (2016) Community-acquired pneumonia caused by Mycoplasma pneumoniae: how physical and radiological examination contribute to successful diagnosis. Front Med 3:28. https://doi. org/10.3389/fmed.2016.00028

Larralde O, Petrik J (2017) Phage-displayed peptides that mimic epitopes of hepatitis E virus capsid. Med Microbiol Immunol 206:301-309. https://doi.org/10.1007/s00430-017-0507-0

Lebreton A et al (2015) Computer-predicted peptides that mimic discontinuous epitopes on the A2 domain of factor VIII. Haemophilia 21:e193-e201. https://doi.org/10.1111/hae.12575

Lee SC, Youn YS, Rhim JW, Kang JH, Lee KY (2016) Early serologic diagnosis of Mycoplasma pneumoniae pneumonia: an observational study on changes in titers of specific- $\operatorname{IgM}$ antibodies and cold agglutinins. Medicine 95:e3605. https://doi.org/10.1097/ md.0000000000003605

Lee WJ et al (2017) Role of serum Mycoplasma pneumoniae IgA, $\mathrm{IgM}$, and $\mathrm{IgG}$ in the diagnosis of Mycoplasma pneumoniae-related Pneumonia in school-age children and adolescents. Clin Vaccine Immunol. https://doi.org/10.1128/cvi.00471-16

Li W, Liu Y, Zhao Y, Tao R, Li Y, Shang S (2015) Rapid diagnosis of Mycoplasma pneumoniae in children with pneumonia by an immuno-chromatographic antigen assay. Sci Rep 5:15539. https ://doi.org/10.1038/srep15539

Li W, Fang YH, Shen HQ, Yang DH, Shu Q, Shang SQ (2017) Evaluation of a real-time method of simultaneous amplification and testing in diagnosis of Mycoplasma pneumoniae infection in children with pneumonia. PLos One 12:e0177842. https://doi.org/10.1371/ journal.pone. 0177842

Liu J et al (2017) B cell cross-epitope of Propionibacterium acnes and Actinobacillus pleuropneumonia selected by phage display library can efficiently protect from Actinobacillus pleuropneumonia infection. Vet Microbiol 205:14-21. https://doi.org/10.1016/j. vetmic.2017.04.026

Muthusamy K, Gopinath K, Nandhini D (2016) Computational prediction of immunodominant antigenic regions \& potential protective epitopes for dengue vaccination. Indian J Med Res 144:587-591. https://doi.org/10.4103/0971-5916.200894

Pinschewer DD (2017) Virally vectored vaccine delivery: medical needs, mechanisms, advantages and challenges. Swiss Med Wkly 147:w14465. https://doi.org/10.4414/smw.2017.14465

Santos PS et al (2012) Functional epitope core motif of the Anaplasma marginale major surface protein 1a and its incorporation onto bioelectrodes for antibody detection. PLoS ONE 7:e33045. https ://doi.org/10.1371/journal.pone.0033045

Singh MK, Shivakumaraswamy S, Gummadi SN, Manoj N (2017) Role of an N-terminal extension in stability and catalytic activity of a hyperthermostable alpha/beta hydrolase fold esterase. Protein Eng Des Sel 30:559-570. https://doi.org/10.1093/protein/gzx049

Smith GP (1985) Filamentous fusion phage: novel expression vectors that display cloned antigens on the virion surface. Science 228(4705):1315-1317

Wang Y, Wang L (2017) Vaccination with phage-displayed antigenic epitope. Methods Mol Biol 1625:225-235. https://doi. org/10.1007/978-1-4939-7104-6_15

Wang L et al (2016) The mimic epitopes of Mycobacterium tuberculosis screened by phage display peptide library have serodiagnostic potential for tuberculosis. Pathog Dis. https://doi.org/10.1093/ femspd/ftw091

Wang L et al (2017) A comparison study between GeXP-based multiplex-PCR and serology assay for Mycoplasma pneumoniae 
detection in children with community acquired pneumonia. BMC Infect Dis 17:518. https://doi.org/10.1186/s12879-017-2614-3

Widjaja M, Berry IJ, Pont EJ, Padula MP, Djordjevic SP (2015) P40 and P90 from Mpn142 are targets of multiple processing events on the surface of Mycoplasma pneumoniae. Proteomes 3:512-537. https://doi.org/10.3390/proteomes3040512

Xiao N, Cao J, Zhou H, Ding SQ, Kong LY, Li JN (2016) Identification of three novel B-cell epitopes of VMH protein from Vibrio mimicus by screening a phage display peptide library. Vet Immunol Immunopathol 182:22-28. https://doi.org/10.1016/j.vetim m.2016.09.005

Xue G, Cao L, Wang L, Zhao H, Feng Y, Ma L, Sun H (2013) Evaluation of $\mathrm{P} 1$ adhesin epitopes for the serodiagnosis of Mycoplasma pneumoniae infections. FEMS Microbiol Lett 340:86-92. https:// doi.org/10.1111/1574-6968.12063

Yang L, Cen J, Xue Q, Li J, Bi Y, Sun L, Liu W (2013) Identification of rabies virus mimotopes screened from a phage display peptide library with purified dog anti-rabies virus serum IgG. Virus Res 174:47-51. https://doi.org/10.1016/j.virusres.2013.02.013

Zeng Y, Liu L, He J, Liu Y, Zhu C, You X, Wu Y (2012) Screening and identification of the mimic epitope of the adhesion protein of Mycoplasma genitalium. Can J Microbiol 58:898-908. https://doi. org/10.1139/w2012-057

Zhou XL et al (2016) Translational quality control by bacterial threonyl-tRNA synthetases. J Biol Chem 291:21208-21221. https:// doi.org/10.1074/jbc.M116.740472 